\title{
Different Roles for Amygdala Central Nucleus and Substantia Innominata in the Surprise-Induced Enhancement of Learning
}

\author{
Peter C. Holland and Michela Gallagher \\ Department of Psychological and Brain Sciences, Johns Hopkins University, Baltimore, Maryland 21218
}

\begin{abstract}
Within most modern learning theories, the discrepancy between expected and obtained outcomes ("prediction error" or "surprise") is a critical determinant of the acquisition of learned associations. The results of studies from many laboratories show that the surprising omission of an expected event may enhance attention to stimuli that remain present, such that subsequent learning about those stimuli is enhanced. A series of reports from our laboratories demonstrated that these surprise-induced enhancements of stimulus associability depend on circuitry that includes the amygdala central nucleus (CeA), the cholinergic neurons in the sublenticular substantia innominata/nucleus basalis magnocellularis (SI/nBM), as well as certain cortical projections of these latter neurons. In this study, we found very different roles for $\mathrm{CeA}$ and SI/nBM in surprise-induced enhancements of stimulus associability. In four experiments that used transient inactivation techniques, we found that surprise-induced enhancement of subsequent learning about a stimulus depended on intact CeA function at the time of surprise but not when more rapid learning was subsequently expressed. In contrast, normal SI/nBM function was critical to the expression of enhanced learning but was not necessary when surprise was induced. These data suggest that these two components of the so-called "extended amygdala" serve distinct roles in the encoding and retrieval of information used in modulating attention to stimuli in associative learning. Additional circuitry linking these brain regions may also be important in the maintenance of that information.
\end{abstract}

Key words: amygdala central nucleus; attention; conditioning; prediction error; substantia innominata; transient inactivation; rat

\section{Introduction}

According to most modern theories of associative learning, learning is enhanced when expectancies are violated (Schultz and Dickinson, 2000). Although the most common instantiation of this claim is that surprising reinforcers are more effective than expected ones (Rescorla and Wagner, 1972), many theorists have asserted that the induction of surprise ("prediction error") in a learning trial enhances attention to potential cues present on that trial. For example, Pearce and Hall (1980) maintained that the magnitude of the prediction error on a conditioning trial determines the learning rate parameter (termed "associability") for the conditioned stimuli present on that trial. Thus, as learning proceeds and the reinforcer becomes better predicted, the conditioned stimuli associability will decline, and subsequent learning about that conditioned stimulus (CS) will be slowed. Likewise, increases in the prediction error produced by omitting an expected event or presenting an unexpected event enhance the associability of the CS and hence facilitate subsequent learning about that event. These predictions are supported by the results of many behavioral experiments (Holland, 1997).

In several behavioral training protocols, we (Holland and Gal-

Received Jan. 27, 2006; revised Feb. 21, 2006; accepted Feb. 23, 2006.

This work was supported in part by National Institutes of Health Grant MH53667. We thank Ezequiel Galarce, Weidong Hu, Erin Kerfoot, Vanessa McKenna, and Anjana Muralidharan for assistance.

Correspondence should be addressed to Dr. Peter C. Holland, Johns Hopkins University, 222 Ames Hall, 3400 North Charles Street, Baltimore, MD 21218. E-mail: pch@jhu.edu.

DOI:10.1523/JNEUROSCI.0390-06.2006

Copyright $\odot 2006$ Society for Neuroscience $\quad$ 0270-6474/06/263791-07\$15.00/0 lagher, 1999) found that enhanced learning about conditioned stimuli after omission of surprising events depends on circuitry that includes the amygdala central nucleus (CeA), the cholinergic substantia innominata/nucleus basalis magnocellularis (SI/ $\mathrm{nBM}$ ), and the posterior parietal cortex (PPC). We proposed that surprise-induced cortical processing of sensory events was enhanced by activity of the SI/nBM, which in turn was modulated by the CeA.

In one of the protocols used in our previous research (Wilson et al., 1992) (Table 1), rats were first extensively exposed to a light $\rightarrow$ tone sequence. Later, rats in a "consistent" condition received additional light $\rightarrow$ tone training, whereas the tone was omitted on one-half of the trials for rats in a "surprise" condition. Within the Pearce and Hall (1980) model, as the light becomes a better predictor of the tone, its associability will decline, but the surprising omission of the tone in the surprise condition will increase its associability. We assessed these associability changes by examining the acquisition of conditioned responses (CRs) to the light when it was paired with food in a final test phase. Intact rats exposed to the surprise treatment learned more rapidly than those that received the consistent treatment. No such effect was observed in rats with bilateral lesions of either the CeA (Holland and Gallagher, 1993) or the SI/nBM (Chiba et al., 1995). This deficit was also displayed by rats in which communication between those two structures was disrupted (Han et al., 1999) and by rats with lesions that removed the cholinergic innervation of the PPC from the SI/nBM (Bucci et al., 1998).

In the present study, we used this protocol to further explore 
Table 1. Outline of behavioral treatment procedures

\begin{tabular}{llll}
\hline Group & Phase 1 & Phase 2 & Test \\
\hline Consistent & Light $\rightarrow$ tone $\rightarrow$ food & Light $\rightarrow$ tone $\rightarrow$ food & Light $\rightarrow$ food \\
& Ligh $\rightarrow$ tone $\rightarrow$ nothing & Light $\rightarrow$ tone $\rightarrow$ nothing & \\
Surprise & Ligh $\rightarrow$ tone $\rightarrow$ food & Light $\rightarrow$ tone $\rightarrow$ food & Light $\rightarrow$ food \\
& Light $\rightarrow$ tone $\rightarrow$ nothing & Light $\rightarrow$ nothing & \\
\hline
\end{tabular}

The surprising omission of the tone (bold 'nothing') on nonreinforced trials in phase 2 in the surprise group is intended to enhance the associability of the light in that group. The associability of the light is assessed by examining the rate of learning of light-food associations in the test phase.

the roles of the CeA and the SI/nBM in these surprise-induced enhancements of attention, by examining the effects of transient inactivation of the CeA or SI/nBM only at the time of surprise or at the time of testing. Thus, we considered whether these regions were critical to the initial processing of the prediction error to alter the associability of the light, the maintenance of that information, or to the later expression of this enhanced associability as more rapid learning.

\section{Materials and Methods}

This study was conducted as four experiments, each with five replications. Each experiment used identical behavioral training procedures but differed in lesion/cannula placement and in the training phase in which neural function was disrupted by infusion of an inactivating agent. In experiments 1 and 2, we targeted CeA function, and in experiments 3 and 4 , we targeted SI/nBM function. In experiments 1 and 3, we examined the effects of inactivation at the time of surprise (phase 2), whereas in experiments 2 and 4, we examined the effects of inactivation at the time of testing.

Inactivation was produced by infusions of 1,2,3,4-tetrahydro-6-nitro2,3-dioxo-benzo[f] quinoxaline-7-sulfonamide (NBQX), a competitive antagonist at ionotropic AMPA/kainate receptors that effectively blocks the induction of EPSCs. This agent has been used extensively in the CeA (Walker and Davis, 1997; McDannald et al., 2004; Groshek et al., 2005). In the SI/nBM, it is notable that Ikonomovic et al. (2000) reported that $97 \%$ of neurons that labeled for the $192 \mathrm{IgG}$-positive receptor [a population of cholinergic neurons critical to performance in the behavioral task used here (Chiba et al., 1995)] also labeled for the AMPA receptor subunit GluR1.

In these experiments, we used a combination of unilateral lesion and inactivation, rather than bilateral infusions of the inactivating agent, to better constrain localization of function. Thus, the rats received a unilateral lesion of the region of interest combined with a contralateral placement of a cannula positioned immediately dorsal to that region. Although the boundaries of the inactivating effects of NBQX cannot be specified, the placement and boundaries of the unilateral lesion are readily defined. Whereas bilateral lesions of either of these target structures produce pronounced impairments, previous studies (Han et al., 1999) showed relatively small effects of unilateral lesions of either the CEA or SI/nBM on performance in this task, even when those unilateral lesions were very large. If the target structure is critical to performance, then rats that receive infusions of NBQX contralateral to a lesion should show significantly poorer performance than those that receive infusions of vehicle. Thus, the observation of limited, specifiable damage on the lesioned side constrains localization of function in this preparation even in the absence of a precise demarcation for the inactivating effects of NBQX.

Subjects. The subjects were 179 male Long-Evans rats (Charles River Laboratories, Raleigh, NC), which weighed $300-325 \mathrm{~g}$ when they arrived in the laboratory vivarium. After 1 week with ad libitum access to food and water, the rats received surgery, followed by $10-14 \mathrm{~d}$ of recovery. They were then reduced to $85 \%$ of their ad libitum weights by restricting their access to food. Throughout the study, the rats lived in individual cages in a colony room illuminated from 6 A.M. to 8 P.M.

Apparatus. The behavioral training apparatus consisted of eight individual chambers $(22.9 \times 20.3 \times 20.3 \mathrm{~cm})$ with aluminum front and back walls, clear acrylic sides and top, and a floor made of $0.48 \mathrm{~cm}$ stainless steel rods spaced $1.9 \mathrm{~cm}$ apart. A dimly illuminated food cup was recessed in the center of the front wall. An infrared photocell placed just inside the food cup was polled $(1 \mathrm{kHz})$ by computer circuitry to record the time each rat spent with its head in the food cup. Each chamber was enclosed in a sound-resistant shell. A speaker, used to present auditory conditioned stimuli, was mounted on the inside wall of the inner shell, $10 \mathrm{~cm}$ above the experimental chamber and even with the front wall of the chamber. A $6 \mathrm{~W}$ lamp was mounted behind a jeweled lens on the front wall, $10 \mathrm{~cm}$ above the food cup; illumination of this lamp served as the light CS. Ventilation fans provided masking noise ( $70 \mathrm{~dB})$. Constant dim illumination was provided by a $6 \mathrm{~W}$ lamp behind a dense red lens mounted next to the speaker. A television camera was mounted within each shell to provide a view of the chamber; the output from each camera was digitized, merged into a single image of all four chambers, and recorded on videotape. Results of behavioral analysis from these videotapes are not reported here, except in passing.

Surgical procedures. Surgery was performed under isoflurane anesthesia with aseptic conditions. In experiments $1(n=51)$ and $2(n=43)$, all rats received unilateral ibotenic acid lesions of the CeA and a contralateral placement of a cannula dorsal to the CeA. For one-half of the rats, the lesions were on the left side, and for the other half, they were on the right side. CeA lesions were made using stereotaxic coordinates $2.4 \mathrm{~mm}$ posterior to bregma and $4.3 \mathrm{~mm}$ from the midline, with infusions at a depth of $7.9 \mathrm{~mm}$ from the skull surface. Each lesion was made using $0.25 \mu \mathrm{l}$ of $10 \mu \mathrm{g} / \mu \mathrm{l}$ ibotenic acid (Sigma, St. Louis, MO) in PBS solution, infused with a $2.0 \mu \mathrm{l}$ Hamilton syringe over a 6 min period. A 31 gauge guide cannula, fitted with a dummy injector (Plastics One, Roanoke Rapids, VA), was chronically implanted in each rat using the same stereotaxic coordinates as were used for the lesion, with the tip at a depth of $5.9 \mathrm{~mm}$ from the skull surface. Lesion and cannula implantation procedures were identical for experiments $3(n=48)$ and $4(n=43)$, except that lesions and cannulas were directed to the SI/nBM. SI/nBM lesions were made using stereotaxic coordinates $0.8 \mathrm{~mm}$ posterior to bregma and 2.3 and 3.3 $\mathrm{mm}$ from the midline, with infusions at depths of -7.8 and $-8.1 \mathrm{~mm}$, respectively. For each of the two infusion sites, $0.2 \mu \mathrm{l}$ of $0.26 \mu \mathrm{g} / \mu \mathrm{l}$ 192IgG-saporin (Chemicon, Temecula, CA) in PBS solution was infused with a $2.0 \mu \mathrm{l}$ Hamilton syringe over a $4 \mathrm{~min}$ period. A guide cannula was implanted contralaterally at the coordinates $0.8 \mathrm{~mm}$ posterior to bregma and $2.8 \mathrm{~mm}$ from the midline, with the tip at a depth of $5.9 \mathrm{~mm}$ from the skull surface. After surgery, all rats received a single $0.02 \mathrm{ml}$ subcutaneous injection of buprenorphine hydrochloride (Sigma) for amelioration of pain and were allowed to recover from surgery for 10-14 $\mathrm{d}$ before additional behavioral testing.

Histological procedures. After completion of behavioral testing, the rats were deeply anesthetized with isofluorane and perfused with $0.1 \mathrm{M}$ PBS, followed by $4 \%$ cold paraformaldehyde. After removal of the cannulas, the brains were removed and stored in $0.1 \mathrm{M}$ PBS with $20 \%(\mathrm{w} / \mathrm{v})$ sucrose at $4^{\circ} \mathrm{C}$ for $24-48 \mathrm{~h}$ and frozen for later analysis. Four series of $40 \mu \mathrm{m}$ sections were taken from each brain, and every fourth section was mounted on slides and Nissl stained to verify the cannula placements and CeA lesions. In experiments 3 and 4, the remaining series were mounted on slides and processed for choline acetyl transferase (ChAT), acetylchoinesterase (AChE), and parvalbumin, using procedures reported previously (Chiba et al., 1995) to more thoroughly characterize the effects of the cholinergic lesions of the SI/nBM. Estimates of CeA lesion sizes were made by outlining lesions on Nissl-stained coronal sections at three anteroposterior levels onto atlas plates. SI lesions were evaluated by qualitative comparisons of lesion and cannula sides on ChAT- and parvalbumin-stained sections and by lesion versus cannula side comparisons of the optical densities of AChE-stained coronal sections of the cortex at three anteroposterior planes.

Infusion procedures. During the last $2 \mathrm{~d}$ of the surgical recovery period, the dummy cannula injectors were removed and replaced to acclimate the rats to manipulation of the cannula headset. In all four experiments, all rats received an infusion of PBS vehicle before each of phase 1 sessions $6-10$. The purpose of these infusions was to familiarize all rats with the infusion procedure, well before surprise was induced in phase 2 . In experiments 1 and 3, before each of the sessions of phase 2, one-half of the rats in each behavioral training group (surprise or consistent) were in- 
fused with NBQX, and one-half were infused with PBS vehicle. In the test phase, all of these rats received PBS infusions before each session. In experiments 2 and 4, all of the rats received PBS infusions before each phase 2 session, but before each of the test sessions, one-half of the rats in each training group received NBQX infusions and one-half received PBS infusions.

Twenty minutes before each designated training session, the dummy cannula was removed from each rat, and an injector cannula was inserted and secured into the guide cannula so that its tip projected $2 \mathrm{~mm}$ beyond the guide cannula. The injector cannulas were connected by PE50 tubing to $10 \mu \mathrm{l}$ Hamilton syringes placed in a multigang precision syringe pump. The syringe pump delivered $0.2 \mu \mathrm{l}$ of $20 \mathrm{mg} / \mathrm{ml} \mathrm{NBQX}$ (Sigma) or the PBS vehicle alone over a $1 \mathrm{~min}$ interval. The injector was left in place for an additional $1 \mathrm{~min}$. After removal of the injector cannula and replacement of the dummy cannula, each rat was placed in a small $(22 \times$ $11 \times 11 \mathrm{~cm}$ ) confinement cage for the time remaining before the behavioral training session.

Behavioral training procedures. Table 1 provides an outline of the procedures that were used in all four experiments. The rats first received two $64 \mathrm{~min}$ sessions to train the rats to approach and eat from the food cup on food delivery. Each of these sessions included 16 deliveries of two food pellets (45 mg grain pellets; Research Diets, New Brunswick, NJ), the reinforcer used throughout these studies. In the next 10 phase 1 training sessions, each rat received 16 presentations of a serial compound stimulus, which comprised a $10 \mathrm{~s}$ illumination of the panel light, followed immediately by a $10 \mathrm{~s}, 78 \mathrm{~dB}, 1500 \mathrm{~Hz}$ tone. One-half of these trials were reinforced with food, and the other half was not. The two types of trials were intermixed randomly, with intertrial intervals that ranged from 2 to $6 \mathrm{~min}$. In phase 2, one-half of the rats in each experiment were assigned to the consistent group. These rats received two additional sessions identical to those of phase 1 . The remaining rats were assigned to the surprise group; these rats received sessions similar to those of the consistent group, except that the nonreinforced light $\rightarrow$ tone trials were replaced by light-alone trials. Thus, the rats in the surprise group would be surprised by the omission of the tone on light-alone trials. The effects of this surprise on the rate of learning about the light was assessed in a final test phase, in which all rats received eight light $\rightarrow$ food pairings in each of three 64 min sessions.

Notably, this training protocol has been used extensively (Wilson et al., 1992; Holland and Gallagher, 1993; Chiba et al., 1995; Bucci et al., 1998) to isolate surprise-induced enhancements of attention apart from other learning processes such as, for example, those specified by the Rescorla and Wagner (1972) model. First, only the predictability of the light $\rightarrow$ tone relationship was altered in the surprise condition; the probability of food delivery on trials with the target light cue remained at 50\% throughout the training phases in both treatment conditions. Thus, rats in both the consistent and surprise conditions had equal opportunities for context conditioning by the food reinforcer or frustration because of its omission. Second, the training conditions were designed to minimize direct light-food conditioning during phases 1 and 2 . The light was placed in an unfavorable temporal relationship with food, relative to the tone-food relationship, and the light was also chosen to be less salient than the tone. Third, these same training conditions have been shown to enhance both excitatory light $\rightarrow$ food learning and inhibitory light $\rightarrow$ no food learning in test (Holland et al., 2001). Thus, with these training conditions, the test procedure reveals attention to the light (as indexed by learning rate) rather than the establishment of previous light-food associations. Holland et al. (2002) and Holland and Gallagher (1993) discuss these issues in more detail.

Behavioral measures. The primary measure of learning was the percentage of time the rat spent in the recessed food cup during the conditioned stimuli, as measured by the infrared photocell sampling system. Because food cup entry is more frequent during the second half of $10 \mathrm{~s}$ conditioned stimuli (Holland, 1977; Gallagher et al., 1990) and this interval is less contaminated by conditioned orienting behaviors (not reported here), we reported food cup responding in the last $5 \mathrm{~s}$ of CS periods.
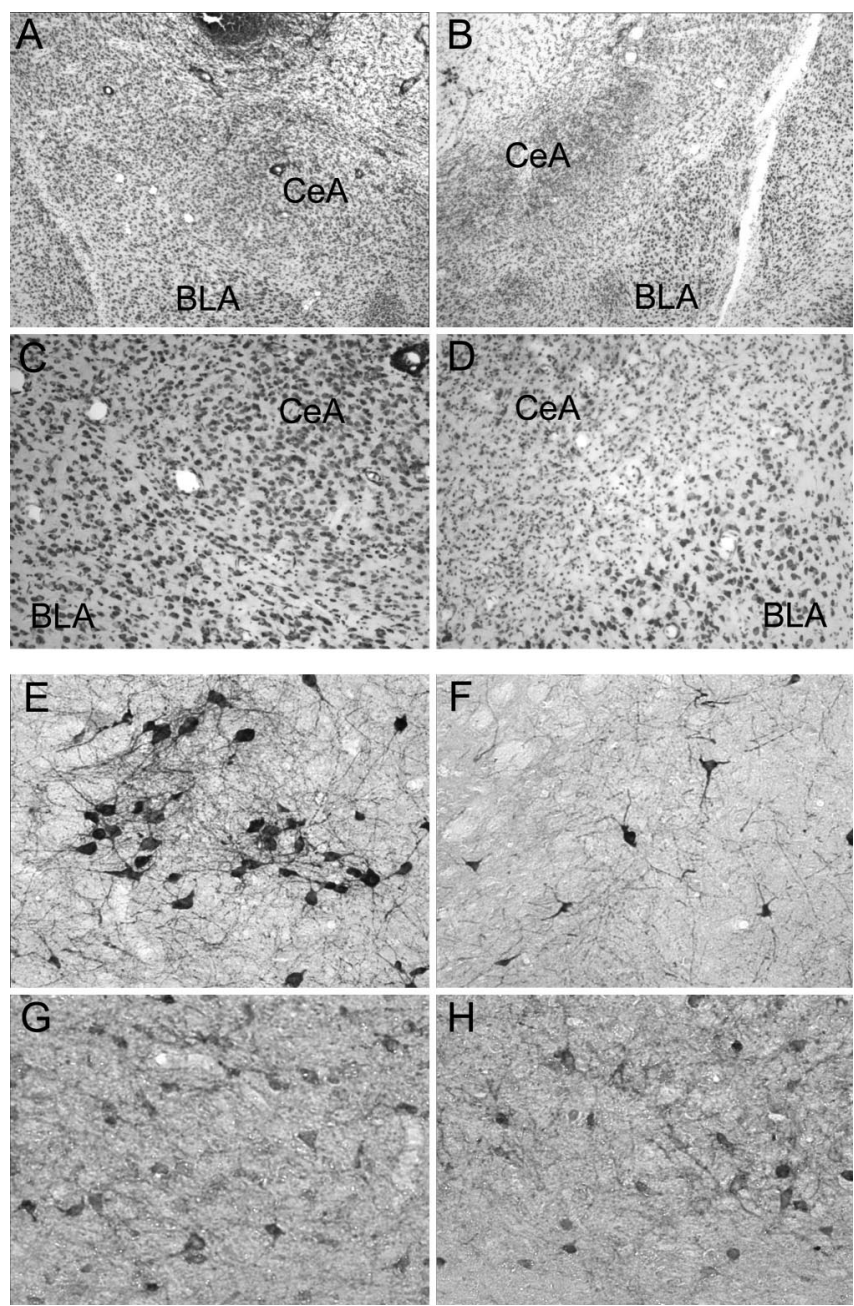

Figure 1. Photomicrogaphs of representative brain sections in experiments 1 and $2(\boldsymbol{A}-\boldsymbol{D})$ and experiments 3 and $4(\boldsymbol{E}-\boldsymbol{H}) . \boldsymbol{A}, \boldsymbol{B}$, Low-power images of the CeA on the cannula $(\boldsymbol{A})$ and lesion $(\boldsymbol{B})$ sides of the same section. Damage from the cannula is visible at the top of $\boldsymbol{A}$, but neurons in the $\mathrm{Ce} A$ are intact. A lesion of the $\mathrm{Ce} A$, with substantial gliosis in the medial region and cell loss along the border with the BLA, is shown in $\boldsymbol{B}$. $\boldsymbol{C}, \boldsymbol{D}$, Higher-power images of those same sections, along the CeA-BLA border. $E, F$, High-power images of ChAT-positive neurons in comparable portions of the $S \mathrm{I} / \mathrm{nBM}$ in the cannula $(\boldsymbol{E})$ and lesioned $(\boldsymbol{F})$ sides of the same section. The density of large cholinergic neurons is greater on the cannula side. $\boldsymbol{G}, \boldsymbol{H}$, Cannula and lesion sides of the parvalbumin-stained section adjacent to the ChAT-stained section shown in $\boldsymbol{E}$ and $\boldsymbol{F}$; the density of parvalbumin-positive GABAergic neurons is comparable in the two sides.

\section{Results}

\section{Histological results}

Of the 94 rats in experiments 1 and 2, 5 died before completion of the study, and 18 were discarded because they lost their headsets, developed obstructed cannulas, had unacceptably small CeA lesions, or excessive damage to the CeA in the hemisphere with the cannula. Lesions were rejected if there was $<40 \%$ damage to the medial portion of the CeA (which projects to the SI/nBM) or if there was more than minimal damage to regions adjoining the CeA. Figure $1 A-D$ shows a Nissl-stained section of a brain with a cannula placement and intact CeA on the right side and a CeA lesion on the left side. The acceptable lesions averaged $61.0 \pm$ $3.3 \%$ damage to the medial $\mathrm{CeA}$ and $41.0 \pm 2.8 \%$ damage to the CeA overall in experiment 1 and $62.0 \pm 3.7 \%$ and $44.3 \pm 3.5 \%$ damage, respectively, in experiment 2 . Neither damage from lesions nor damage from cannulas extended to the SI/nBM in any brain. Approximately equal numbers of rats with acceptable le- 
sions were found in each of the four combinations of the behavioral training group (consistent or surprise) and infusion (NBQX or vehicle) during the critical phase (Table 2). Furthermore, within each of these conditions, approximately equal numbers of rats had cannulas on each side.

Of the 85 rats in experiments 3 and 4, 2 died before completion of the study, and 17 were excluded because they lost their headsets, developed obstructed cannulas, had unacceptably small SI/nBM lesions, or had more than minimal damage to the SI/ $\mathrm{nBM}$ in the hemisphere with the cannula. All rats showed substantial damage to the lateral globus pallidus on the cannula side but no such damage contralaterally. The acceptable lesions were characterized by substantial loss of ChAT-positive magnocellular neurons in the SI/nBM region (Fig. $1 E, F$ ) and by lighter AChE staining throughout the neocortex, relative to the unlesioned (cannula) side. Importantly, the lesions in experiments 3 and 4 were comparable. The cortical optical densities were $24.4 \pm 2.5 \%$ lower on the lesioned side than on the cannula side in experiment 3 and $24.8 \pm 1.6 \%$ lower in experiment 4 . Within each experiment, approximately equal numbers of rats with acceptable lesions were found in each of the four combinations of the behavioral training group (consistent or surprise) and infusion (NBQX or vehicle) during the critical phase (Table 2). Furthermore, within each of these conditions, approximately equal numbers of rats had cannulas on each side.

The lesions did not extend into the medial septal area or the vertical limb of the diagonal band, and in many of the brains, there was a relatively small loss of neurons in the horizontal limb of the diagonal band. Furthermore, parvalbumin immunostaining revealed no hemispheric differences (i.e., lesion or cannula) in the distribution of GABAergic neurons within the SI/nBM region (Fig. $1 G, H$ ). Damage from either lesions or cannulas did not extend into the CeA in any brain in experiments 3 and 4 .

\section{Behavioral results}

Eleven rats in experiments 1 and 2 and four rats in experiments 3 and 4 failed to acquire conditioned responding to the serial compound cues by the end of phase 1 , spending $<10 \%$ of their time in the food cup during the tone. These rats were discarded. Because within each pair of experiments all of the rats had been treated identically up to that point in the study, this loss of subjects was not attributable to differences in the treatment or infusion condition.

In phase 1, the remaining rats in all four experiments rapidly acquired moderate levels of conditioned responding to the tone, which immediately preceded food delivery, and low levels of responding to the visual CS, which was more remote from the food. Table 2 shows the levels of responding attained by the last two sessions of phase 1 in each of the four treatment conditions in each experiment. For each experiment, group (consistent or surprise) by subsequent infusion (NBQX or vehicle to be infused in later phases) by session (1-10) ANOVAs for each stimulus period (light, tone, and pre-CS periods) showed no significant effects of either group or subsequent infusion $(p>0.153)$ nor interactions of those variables with the other variables $(p>0.164)$. Group- by-infusion ANOVAs for the final two sessions combined yielded comparable results. Thus, in each experiment, the rats entered phase 2 with similar response levels in all treatment conditions.

In phase 2, the tone was omitted on nonreinforced trials in the surprise groups in all experiments. Tone omission had no effect on performance during light $\rightarrow$ tone $\rightarrow$ food trials (common to all groups) in any experiment (Table 2); separate treatment-byinfusion ANOVAs for the tone, light, and pre-CS periods in each experiment showed no significant effects of treatment $(F<1 ; p>$ $0.395)$. In experiments 1 and 3 , the rats in the surprise-NBQX and consistent-NBQX groups received intracranial infusions of NBQX before each phase 2 session. In experiment 1 , performance of previously established CRs during these periods was unaffected by infusion of NBQX into the CeA $(F<1$; $p>0.570)$. However, in experiment 3 , although infusion of NBQX into the $\mathrm{SI} / \mathrm{nBM}$ had no significant effect on responding in the light or pre-CS periods $(F<1 ; p>0.580)$, it suppressed responding to the tone (Table 2 ) in both the consistent and surprise treatment conditions $\left(F_{(1,24)}=4.67 ; p=0.041\right)$. This suppression of responding was transient. On the first phase 2 session, responding in the NBQX-infused consistent and surprise groups was reduced to $47.6 \pm 12.1 \%$ and $45.1 \pm 9.9 \%$, respectively, compared with $76.1 \pm 7.8 \%$ and $71.1 \pm 9.9 \%$ in the comparable vehicle-infused groups $\left(F_{(1,24)}=7.61 ; p=0.011\right)$, but on the second phase 2 session, responding to the tone did not differ significantly among the groups (range: $61.4 \pm 14.8$ to $74.1 \pm 9.1 \% ; F_{(1,24)}=2.12 ; p=$ $0.158)$.

Figure 2 shows the primary data from each experiment, the results of the final test phase in which the associability of the light CS was assessed by measuring the acquisition of CRs to that cue when it was paired with food. If omission of the tone in phase 2 in the surprise groups enhanced the associability of the light, then rats in those groups should acquire more conditioned responding over the course of testing than rats in the consistent groups. In all four experiments, vehicle control rats in the surprise group indeed showed more light $\rightarrow$ food learning than those in the consistent group. However, both inactivation of the CeA during the phase 2 surprise sessions (experiment 1) (Fig. 2A) and inactivation of the SI/nBM during the test sessions (experiment 4) (Fig. 

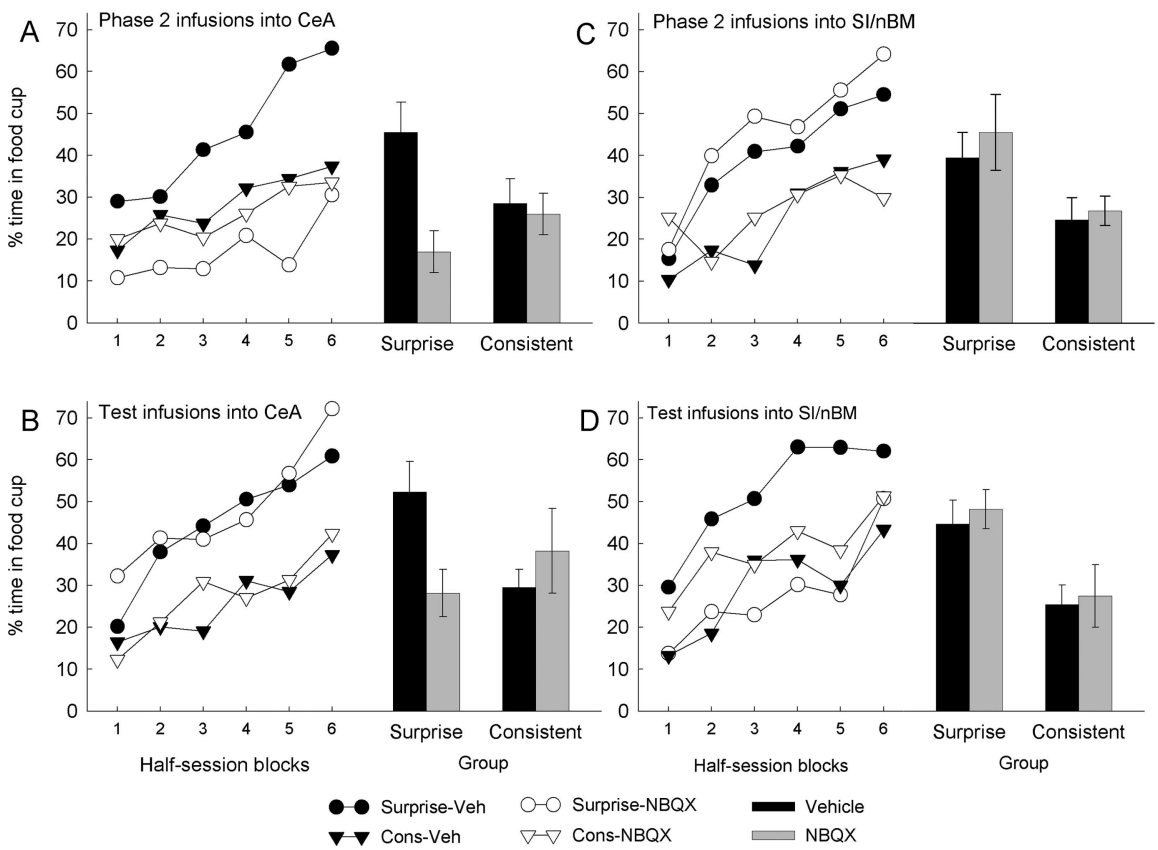

Figure 2. Behavioral data from the test phase of experiments 1-4. The left side of each panel shows the acquisition of food cup CRs over the course of the test phase, and the right side of each panel shows the mean ( \pm SEM) responding over the entire test phase. The target of the lesions and cannulas was the CeA in experiments 1 and $2(\boldsymbol{A}, \boldsymbol{B})$ and the $S I / n B M$ in experiments 3 and $4(\boldsymbol{C}$, $D$ ). The rats in the NBQX subgroups received infusions of NBQX during either the phase 2 surprise treatment (experiments 1 and 3 ; $\boldsymbol{A}, \boldsymbol{C}$ ) or the test phase (experiments 2 and $4 ; \boldsymbol{B}, \boldsymbol{D}$ ); the rats in the vehicle-only (Veh) subgroups received infusions of vehicle alone in those phases. Surprise-induced enhancements in cue associability are reflected in greater responding in the surprise group than in the consistent (Cons) group. Vehicle-only rats showed significant surprise-induced enhancement of learning in all four experiments. This enhancement was eliminated by infusions of NBQX into the CeA during the surprise phase but not the test phase and by infusions of NBQX into the SI/nBM during the test phase but not the surprise phase.

$2 D$ ) eliminated that more rapid learning by rats in the surprise groups. In contrast, neither inactivation of the CeA during the test sessions (experiment 2) (Fig. 2B) nor inactivation of the $\mathrm{SI} / \mathrm{nBM}$ during phase 2 surprise sessions (experiment 3) (Fig. 2C) affected this normal surprise-induced enhancement of learning rate. Thus, CeA function was critical only at the time of surprise, presumably for adjusting the associability of the light cue, whereas SI/nBM function was critical only when that enhanced associability was expressed in more rapid learning.

For each experiment, responding during the light CS was subjected to a group (consistent or surprise) by infusion (NBQX or vehicle) by half-session blocks of trials (1-6) ANOVA. Effects of inactivation specific to surprise-induced enhancement of learning would be revealed by less learning by NBQX-infused rats in the surprise group but no differences attributable to infusion in the consistent group. This pattern of data would be indicated by a significant group-by-infusion interaction and subsequent planned contrasts of responding of NBQX versus vehicle rats in each of the two treatment groups.

In experiment 1 (Fig. 2A), CeA inactivation at the time of surprise (phase 2) eliminated the normal surprise-induced enhancement of subsequent learning in the test phase. The groupby-infusion interaction was significant $\left(F_{(1,26)}=4.93 ; p=0.035\right)$, as were the effects of infusion $\left(F_{(1,26)}=6.92 ; p=0.014\right)$ and blocks $\left(F_{(5,130)}=14.20 ; p<0.001\right)$. Individual contrasts showed that responding of rats in the surprise-NBQX group was significantly lower than that in the surprise-vehicle group $\left(F_{(1,26)}=\right.$ $11.77 ; p=0.002)$, but responding of rats in the consistent-NBQX and consistent-vehicle groups did not differ $(F<1 ; p=0.775)$. Responding in the pre-CS (baseline) intervals ranged from $8.8 \pm$ 1.9 to $16.9 \pm 5.8 \%$; ANOVAs such as those performed for re- sponding during the light CS showed no significant main effects $\left(F_{(1,26)}<1.34 ; p>\right.$ $0.259)$ or group-by-infusion interaction $(F<1 ; p=0.935)$ for that measure.

In experiment 2 (Fig. $2 \mathrm{~B}$ ), CeA inactivation in the test phase did not affect the surprise advantage. The group-byinfusion interaction was not significant $\left(F_{(1,25)}<0.01 ; p=0.972\right)$. At the same time, the effect of group was significant $\left(F_{(1,25)}=9.10 ; p=0.006\right)$, indicating that surprise while CeA function was intact enhanced subsequent learning about the light in the test sessions, regardless of the status of the CeA at the time of that test. NBQX- and vehicle-infused rats in both the surprise and consistent groups performed similarly $(F<1 ; p=0.919$ and 0.762 , respectively). The effect of blocks was also significant $\left(F_{(5,125)}=21.86 ; p<\right.$ 0.001 ). Responding in the pre-CS intervals ranged from $8.7 \pm 2.3$ to $16.8 \pm 4.6 \%$; ANOVAs such as those performed for responding during the light CS showed no significant main effects $(F<1 ; p>0.504)$ or group-by-infusion interaction $\left(F_{(1,25)}=\right.$ $2.62 ; p=0.118)$ for pre-CS responding.

It is notable that the lesions and cannula placements of experiment 2 were indistinguishable from those of experiment 1 , in which inactivation during phase 2 eliminated the surprise effect. An analysis of the acquisition of conditioned orienting responses (ORs; rearing) to the visual CS in the test phase of experiment 2 also confirmed the behavioral efficacy of the lesion/inactivation procedure in this experiment, despite its not affecting the surprise effect. Gallagher et al. (1990) showed that bilateral CeA lesions disrupted the acquisition of conditioned ORs to the onset of visual conditioned stimuli but not the acquisition of learned behaviors directed toward the food cup. Unilateral CeA lesions had no such effect (Han et al., 1997, 1999). An analysis of video recordings of the test sessions of experiment 2 showed substantially less acquisition of rearing in rats that received NBQX infusions in the test than in rats that received vehicle infusions $[12.2 \pm 2.9 \%$ vs $27.2 \pm 3.3 \%$ over the test phase $\left.\left(F_{(1,25)}=13.38 ; p=0.001\right)\right]$.

In experiment 3 (Fig. $2 C$ ), SI/nBM inactivation at the time of surprise (phase 2) did not affect the expression of enhanced learning during the subsequent test phase. The group-byinfusion interaction was not significant $\left(F_{(1,24)}<1\right.$; $\left.p=0.763\right)$. At the same time, the effect of group was significant $\left(F_{(1,24)}=\right.$ $7.14 ; p=0.013)$, indicating that $\mathrm{SI} / \mathrm{nBM}$ function at the time of surprise was irrelevant to the subsequent display of surpriseinduced enhancement of learning. Individual contrasts showed that NBQX- and vehicle-infused rats in both the surprise and consistent groups performed similarly $(F<1 ; p=0.503$ and 0.806 , respectively). The effect of blocks was also significant $\left(F_{(5,125)}=15.20 ; p<0.001\right)$. Responding in the pre-CS intervals ranged from $11.1 \pm 2.5$ to $16.2 \pm 5.8 \%$; ANOVAs such as those performed for responding during the light CS showed no significant main effects $(F<1 ; p>0.533)$ or group-by-infusion interaction $\left(F_{(1,24)}<1 ; p=0.521\right)$ for that measure.

It is notable that the lesions and cannula placements of experiment 3 were indistinguishable from those of experiment 4 , in which 
the infusion of NBQX into the SI in the test phase had significant effects on behavior. Thus, because these lesions and inactivations were behaviorally effective in experiment 4 , the lack of effects in experiment 3 cannot be attributed to the comparatively smaller cholinergic damage produced here than in previous studies in which bilateral lesions were made before all training (Chiba et al., 1995).

Finally, in experiment 4 (Fig. 2 D), SI/nBM inactivation in the test phase eliminated the normal effect of phase 2 surprise. The group-by-infusion interaction was significant $\left(F_{(1,30)}=5.22 ; p=\right.$ $0.030)$, as was the effect of blocks $\left(F_{(5,150)}=19.01 ; p<0.001\right)$. Individual contrasts showed that responding of rats in the surprise-NBQX group was significantly less than that in the surprise-vehicle group $\left(F_{(1,30)}=5.54 ; p=0.025\right)$, but responding of rats in the consistent-NBQX and consistent-vehicle groups did not differ $(F<1 ; p=0.366)$. Responding in the pre-CS intervals ranged from $17.8 \pm 5.8$ to $20.4 \pm 6.4 \%$; ANOVAs such as those performed for responding during the light CS showed no significant main effects $(F<1 ; p>0.712)$ or group-by-infusion interaction $\left(F_{(1,30)}<1 ; p=0.911\right)$ on pre-CS responding.

Although the 192IgG-saporin lesions were specific to a population of magnocellular cholinergic neurons, the contralateral infusion of NBQX is likely to have disrupted the function of a wider range of neuron types. Thus, the effects of NBQX infusion observed in experiment 4 might be attributable to unilateral inactivation of this broader range of neurons rather than to bilateral disruption of cholinergic function alone. However, in this regard, it is worth noting that Chiba et al. $(1994,1995)$ found that lesions of the SI/nBM made with AMPA, which produced much less damage to cholinergic neurons but greater damage to other neuron types than those made with 192IgG-saporin, had smaller effects on performance in the task used here than the latter lesions. Furthermore, in the present study, unilateral NBQX infusion had no effect on performance in rats that were discarded because of a lack of a contralateral SI/nBM lesion. Finally, in a previous study using this procedure, even very substantial unilateral lesions of the SI/nBM had no observable effects (Han et al., 1999). Thus, we think it reasonable to attribute the effects of contralateral SI/nBM 192IgG-saporin lesion/NBQX inactivation to bilateral disruption of cholinergic function.

\section{Discussion}

The results of these experiments indicate that the CeA and SI/ $\mathrm{nBM}$ play distinct roles in surprise-induced enhancement of learning in this task. CeA function must be intact when surprise is induced, but is unimportant at the time of testing for enhanced new learning. Thus, the CeA is critical to the augmentation of CS associability by surprise, or to the processing of that surprise (prediction error) itself. However, once the associability of the CS is adjusted, the expression of more rapid learning to that cue does not depend on CeA function. In contrast, the SI/nBM is not importantly involved in either the processing of the prediction error when it occurs or the consequent changes in CS associability. Nevertheless, SI/nBM function is critical to the expression of more rapid learning once CS associability has been altered (e.g., by modulating activity in the PPC or other cortical regions) (Chiba et al., 1995; Bucci et al., 1998).

Notably, our observations that CeA function is not needed at the time of expression of this function and SI/nBM function is not required at the time of surprise or induction of increases in CS associability constrains identification of likely candidates for the maintenance of information about increased CS associability. If this information were maintained solely in the CeA, then bilateral disruption of CeA function in testing should prevent the expression of previously enhanced CS associability in new learning about the light. No such effect was observed in experiment 2. Likewise, if the CeA acted at the time of surprise to store associability information in the SI/nBM, then functional inactivation of the $\mathrm{SI} / \mathrm{nBM}$ at that time would prevent such storage. No such effect was observed in experiment 3.

One possible resolution of this discrepancy is that alteration of the SI/nBM by the CeA occurs over an extended time period after the surprise sessions themselves, after the inactivating effects of NBQX have dissipated. Many authors have asserted that the consolidation of memories can involve substantial periods of processing after the learning experience (McGaugh et al., 2000; Hoffman and McNaughton, 2002). For example, Marr (1971) suggested that memory storage in cortical regions can be enhanced by rehearsal-like neural processing in the hippocampus that occurs in periods of slow-wave sleep long after training sessions. By analogy, phase 2 transient inactivation of the SI/nBM may have been ineffective in disrupting subsequent enhancements in the learning rate because the CeA continued to "train" the SI/nBM for an extended period of time, after the effects of NBQX dissipated. By this view, a longer-lasting inactivation of the SI/nBM at the time of surprise might produce deficits in subsequent learning.

We consider another account for our data more likely. The CeA may alter an additional brain region at the time of surprise, and that region in turn influences the SI/nBM at the time of expression. In this regard, it is useful to note analogous results from previous studies that showed time-limited roles for the CeA and other brain regions in another attentional function of the $\mathrm{CeA}$, the acquisition of conditioned ORs to conditioned stimuli paired with food delivery. The acquisition and expression of those learned ORs, but not the production of unconditioned ORs or for the acquisition of food-related CRs, depends on the integrity of a circuit that includes the CeA and the dorsolateral striatum (DLS) (Han et al., 1997). As with surprise-induced enhancement of CS associability, CeA function was critical to the acquisition of conditioned ORs but not to the expression of previously acquired ORs (McDannald et al., 2004; Groshek et al., 2005), whereas the opposite was true of the DLS (Han et al., 1997). Thus, in both that case and the results presented in this study, the CeA is critical for the acquisition of an attentional function, conditioned ORs, or enhanced associability but not for its ultimate expression. Similarly, in both cases, structures efferent to the CeA, DLS and SI/nBM, respectively, were critical to the expression of these CeA-dependent functions but were not needed at the time of their acquisition.

In the case of conditioned ORs, CeA communication with the DLS occurs via CeA innervation of the substantia nigra pars compact $(\mathrm{SNc})$, and thus the $\mathrm{SNc}$ was an obvious candidate intermediate structure for storage of information critical to conditioned ORs. Indeed, we found that disconnection of the CeA and SNc prevented the acquisition of conditioned ORs (Lee et al., 2005a), and lesion/inactivation studies such as those performed here showed that intact $\mathrm{SNc}$ function is required at both the time of acquisition and the time of expression (H. El-Amamy and P. C. Holland, unpublished observation). This pattern of data is consistent with the claim that, for learned orienting, the SNc is influenced by the CeA at the time of acquisition and influences the DLS at the time of expression.

Interestingly, recent data suggest that surprise-induced enhancements of learning in the task used here also depend on communication between the CeA and SNc. Lee et al. (2005b) found that rats with contralateral disconnection lesions of the $\mathrm{CeA}$ and $\mathrm{SNc}$ failed to show this enhanced learning, whereas 
normal performance was observed in rats with ipsilateral lesions of these two structures. Notably, SNc dopamine neurons project to SI/nBM (Hasue and Shammah-Lagnado, 2002). Thus, CeA might influence SNc at the time of surprise, and SNc input may, in turn, influence SI/nBM activity at the time of expression of the augmented CS associability. By this view, the direct $\mathrm{CeA} \rightarrow \mathrm{SI}$ projections we proposed previously (Holland and Gallagher, 1999) as mediating this effect may not provide a sufficient basis. Instead, the communication between the CeA and SI/nBM required for surprise-induced enhancements of associability (Han et al., 1999) may include essential indirect components, involving $\mathrm{CeA}-\mathrm{SNc}$ and $\mathrm{SNc}-\mathrm{SI} / \mathrm{nBM}$ projections. This proposed involvement of mid-brain dopamine systems in a circuit critical for the surprise-induced enhancement of CS associability is consistent with the widely appreciated role of dopamine neurons in the generation of prediction error signals (Schultz and Dickinson, 2000). Indeed, consistent with the Pearce and Hall (1980) model, Fiorillo et al. (2003) observed that the sustained activity of dopamine neurons during reward signals was greatest under conditions of maximum uncertainty about the delivery of reward (i.e., when cues were reinforced on $50 \%$ of their trials, as in the present studies).

Mapping of brain systems engaged in particular behavioral tasks requires specification of the component processes that underlie performance in those tasks. The Pearce and Hall (1980) model, for example, assumes both a mechanism for generating prediction error signals and a mechanism by which those signals can modify learning rate parameters (associability) of cues. Previously, we (Chiba et al., 1995; Holland and Gallagher, 1999) suggested that certain instances of surprise-enhanced learning reflected increased cortical processing of cues. Our previous evidence suggested that this increased cortical processing occurs as a result of cholinergic modulation via the SI/nBM under the control of the CeA and SI/nBM. The data of Lee et al. (2005a) suggest that this system for controlling the associability of cues may gain access to prediction error information through its connections with the SNc, which innervates both the CeA (Swanson, 1982) and SI/nBm (Hasue and Shammah-Lagnado, 2002). The present also data show that the roles of the components of this system are time limited (i.e., the role of the $\mathrm{CeA}$ is limited to the time of surprise, whereas the SI/NBM must be active at the time enhanced associability is expressed in more rapid learning).

\section{References}

Bucci DJ, Holland PC, Gallagher M (1998) Removal of cholinergic input to rat posterior parietal cortex disrupts incremental processing of conditioned stimuli. J Neurosci 18:8038-8046.

Chiba AA, Han J-S, Holland PC, Gallagher M (1994) Substantia innominata lesions impair incremental attentional processing in a pavlovian conditioning paradigm. Soc Neurosci Abstr 20:1215.

Chiba AA, Bucci DJ, Holland PC, Gallagher M (1995) Basal forebrain cholinergic lesions disrupt increments but not decrements in conditioned stimulus processing. J Neurosci 15:7315-7322.

Fiorillo CD, Tobler PN, Schultz W (2003) Discrete coding of reward probability and uncertainty by dopamine neurons. Science 299:1898-1902.

Gallagher M, Graham PW, Holland PC (1990) The amygdala central nucleus and appetitive pavlovian conditioning: lesions impair one class of conditioned behavior. J Neurosci 10:1906-1911.

Groshek F, Kerfoot EC, McKenna V, Polackwich AS, Gallagher M, Holland PC (2005) Amygdala central nucleus function is necessary for learning but not expression of conditioned auditory orienting. Behav Neurosci 119:202-212.

Han J-S, McMahan RW, Holland PC, Gallagher M (1997) The role of an amygdalo-nigrostriatal pathway in associative learning. J Neurosci 17:3913-3919.

Han J-S, Holland PC, Gallagher M (1999) Disconnection of amygdala central nucleus and substantia innominata/nucleus basalis disrupts increments in conditioned stimulus processing. Behav Neurosci 113:143-151.

Hasue RH, Shammah-Lagnado SJ (2002) Origin of the dopaminergic innervation of the central extended amygdala and accumbens shell: a combined retrograde tracing and immunohistochemical study in the rat. J Comp Neurol 454:15-33.

Hoffman KL, McNaughton BL (2002) Sleep on it: cortical reorganization after-the-fact. Trends Neurosci 25:1-2.

Holland PC (1977) Conditioned stimulus as a determinant of the form of the Pavlovian conditioned response. J Exp Psychol Anim Behav Proc 3:77-104.

Holland PC (1997) Brain mechanisms for changes in processing of conditioned stimuli in Pavlovian conditioning: implications for behavior theory. Anim Learn Behav 25:373-399.

Holland PC, Gallagher M (1993) Amygdala central nucleus lesions disrupt increments, but not decrements, in CS processing. Behav Neurosci 107:246-253.

Holland PC, Gallagher M (1999) Amygdala circuitry in attentional and representational processes. Trends Cogn Sci 3:65-73.

Holland PC, Chik Y, Zhang Q (2001) Inhibitory learning tests of conditioned stimulus associability in rats with lesions of the amygdala central nucleus. Behav Neurosci 115:1154-1158.

Holland PC, Bashaw M, Quinn J (2002) Amount of training and stimulus salience affects associability changes in serial conditioning. Behav Processes 59:169-183.

Ikonomovic MD, Nocera R, Mizukami K, Armstrong DM (2000) Agerelated loss of the AMPA receptor subunits GluR2/3 in the human nucleus basalis of Meynert. Exp Neurol 166:363-375.

Lee HJ, Groshek F, Cantalini JP, Petrovich GD, Gallagher M, Holland PC (2005a) Role of amygdalo-nigral circuitry in conditioning of a visual stimulus paired with food. J Neurosci 25:3881-3888.

Lee HJ, Youn JM, O MJ, Gallagher M, Holland PC (2005b) Role of amygdala-nigral connections in enhanced attention for conditioned stimulus processing. Soc Neurosci Abstr 35:411.15.

Marr D (1971) Simple memory: a theory for archicortex. Philos Trans R Soc Lond B Biol Sci 262:23-81.

McDannald M, Kerfoot E, Gallagher M, Holland PC (2004) Amygdala central nucleus function is necessary for learning but not expression of conditioned visual orienting. Eur J Neurosci 20:240-248.

McGaugh JL, Ferry B, Vazdarjanova A, Roozendaal B (2000) Amygdala: role in modulation of memory storage. In: The amygdala: a functional analysis, Ed 2 (Aggleton JP, ed), pp 391-423. New York: Oxford UP.

Pearce JM, Hall G (1980) A model for Pavlovian learning: variations in the effectiveness of conditioned but not of unconditioned stimuli. Psychol Rev 106:532 552.

Rescorla RA, Wagner AR (1972) A theory of Pavlovian conditioning: variations in the effectiveness of reinforcement and nonreinforcement. In: Classical conditioning II (Black AH, Prokasy WF, eds), pp 64-99. New York: Appleton-Century-Crofts.

Schultz W, Dickinson A (2000) Neuronal coding of prediction errors. Annu Rev Neurosci 23:473-500.

Swanson LW (1982) The projections of the ventral tegmental area and adjacent regions: a comobined fluorescent retrograde tracer and immunofluorescence study in the rat. Brain Res Bull 9:321-333.

Walker DL, Davis M (1997) Double dissociation between the involvement of the bed nucleus of the stria terminalis and the central nucleus of the amygdala in startle increases produced by conditioned versus unconditioned fear. J Neurosci 17:9375-9583.

Wilson PN, Boumphrey P, Pearce JM (1992) Restoration of the orienting response to a light by a change in its predictive accuracy. Q J Exp Psychol 44B:17-36. 